\title{
Spreading Depolarizations and Subarachnoid Hemorrhage
}

\author{
Kazutaka Sugimoto $^{1,2}$ - David Y. Chung ${ }^{1,3,4}$
}

Published online: 22 April 2020

(C) The American Society for Experimental NeuroTherapeutics, Inc. 2020

\begin{abstract}
Cortical spreading depolarizations (SD) are strongly associated with worse tissue injury and clinical outcomes in the setting of aneurysmal subarachnoid hemorrhage (SAH). Animal studies have suggested a causal relationship, and new therapies to target SDs are starting to be tested in clinical studies. A recent set of single-center randomized trials assessed the effect of the phosphodiesterase inhibitor cilostazol in patients with SAH. Cilostazol led to improved functional outcomes and SD-related metrics in treated patients through a putative mechanism of improved cerebral blood flow. Another promising therapeutic approach includes attempts to block SDs with, for example, the NMDA receptor antagonist ketamine. SDs have emerged not only as a therapeutic target but also as a potentially useful biomarker for brain injury following SAH. Additional clinical and preclinical experimental work is greatly needed to assess the generalizability of existing therapeutic trials and to better delineate the relationship between SDs, SAH, and functional outcome.
\end{abstract}

Keywords Cortical spreading depressions · Delayed cerebral ischemia · Vasospasm · Delayed ischemic neurological deficits and stroke $\cdot$ Cerebral aneurysm

\section{Introduction}

Progressive brain injury and poor neurocognitive outcomes following aneurysmal subarachnoid hemorrhage (SAH) remain important problems in survivors of the disease [1]. Determinants of these outcomes are ill-defined because of the difficulty in characterizing the syndromes of early brain injury [2] and delayed cerebral ischemia after SAH [3], but likely involve a combination of large- and small-vessel

Electronic supplementary material The online version of this article (https://doi.org/10.1007/s13311-020-00850-5) contains supplementary material, which is available to authorized users.

David Y. Chung

dychung@mgh.harvard.edu

1 Neurovascular Research Unit, Department of Radiology, Massachusetts General Hospital, Harvard Medical School, 149 13th Street, 6403, Charlestown, MA 02129, USA

2 Department of Neurosurgery, Yamaguchi University School of Medicine, Ube, Japan

3 Division of Neurocritical Care, Department of Neurology, Boston Medical Center, Boston, MA, USA

4 Division of Neurocritical Care, Department of Neurology, Massachusetts General Hospital, Harvard Medical School, Boston, MA, USA vasospasm, microthrombotic events, and inflammation [4, 5]. Furthermore, clinical studies consistently show a strong association between the phenomenon of spreading depolarization (SD) and worse outcomes following SAH [6].

$\mathrm{SD}$ is characterized by a massive depolarization of all cell types and spreads through the cortex by contiguity with surrounding brain tissue [7]. SDs are implicated in multiple diseases ranging from migraine aura to acute brain injury like $\mathrm{SAH}$, intracerebral hemorrhage, subdural hematoma, and brain trauma and result in a very broad spectrum of clinical symptoms [8]. Therefore, SDs occur along a continuum from short-lasting harmless to terminal deleterious events, all of which are observed in patients with $\mathrm{SAH}$ [9]. In injured brain following $\mathrm{SAH}$, they can lead to vasoconstriction and secondary injury through mechanisms involving decreased blood flow, increased energy demand, cytokine release, and blood-brain barrier breakdown. SDs have also been implicated as a mechanism underlying cytotoxic, ionic, and vasogenic edema [10-13]. Emerging evidence has implicated a role for SD in facilitating water influx from the cerebrospinal compartment into the tissue along the glymphatic pathway [14]. The network of factors involving SDs and further injury following SAH presents potential opportunities for therapeutic intervention.

This review will focus on clinical-translational and experimental lab-based evidence on the relationship between SDs and SAH. An overview of approaches to animal modeling of 
SAH alone has been recently described elsewhere [4]. We will take a bedside-to-bench-to-bedside approach in describing clinical observations, animal studies, and finally therapeutic targeting of SDs in patients with SAH.

\section{Methods}

We performed a search in PubMed encompassing dates up until April 24, 2019, for studies investigating SD in relation to SAH, with the following keywords (subarachnoid hemorrhage OR delayed ischemic neurological deficits OR delayed cerebral ischemia) AND (spreading depolarization OR spreading depolarizations OR spreading depression OR CSD OR spreading depressions).

Any observational or experimental studies on spreading depolarization were eligible if they reported on SD in relation to SAH. Both human studies and in vivo or in vitro animal studies were eligible. We excluded reviews and articles not published in English. We further checked the bibliographies of the captured articles for potential studies missed by the database search. Finally, we allowed for additional articles to be added during manuscript preparation and peer review.

\section{Results}

We identified 186 articles in PubMed. After screening the titles and abstracts for eligibility, we retrieved 143 articles. We then excluded articles that were reviews, those that were not in English, and those that did not attempt to detect spreading depolarizations. There were 40 articles included in the initial search, with 3 additional articles from 2019 or appropriate for inclusion but missed on our initial search that were added during manuscript preparation and peer review [15-17]. Otherwise, the studies were published between 1971 and 2018. We organized the studies into 3 groups: clinical observational, animal, and clinical treatment studies.

\section{Clinical Observational Studies}

\section{SDs Are Associated with Worse Outcomes in SAH Patients}

Our search captured 20 papers in which SDs were detected in patients with SAH (Table 1). The majority of the studies were prospective and included patients implanted with subdural electrodes, which remain the gold standard to detect SDs. A landmark study published in 2006 established the clinical association between SD and high-grade SAH and found that $72 \%$ of examined patients had SDs [18]. The authors describe several cases in detail and found a dose dependence between the duration of subsequent depression of brain activity and the presence of delayed infarcts. A subsequent study looked specifically at the impact of SD clusters (please see Glossary for definitions) - as opposed to a single SD in isolation - and found that prolonged depression during SD clusters is associated with progressive damage [19]. The effect of prolonged depressions on worse outcome was confirmed in additional studies [21,23], one of which also found an association between SDs and the development of late epilepsy [21]. Independent of post-SD depression duration, investigators also determined that a longer duration of the SD itself was associated with DCI [22].

Additional studies have examined the relationship between SDs and DCI [20] and early brain injury (EBI) [17, 24]. A case series found that SDs were associated with DCI independent of angiographic vasospasm [20]. This study is distinguished by addressing a key controversy in the SAH field (i.e., is angiographic vasospasm necessary for DCI?), mitigated angiographic vasospasm as a confounder by treating all patients with surgically implanted vasodilator pellets, and focused on the interaction between SDs and DCI in the pathogenesis of secondary injury after SAH. Another study characterized the electrophysiological morphology of the SD and found an association not just with development of DCI but also with early brain injury following SAH [24]. These and prior studies looked at temporal associations between SDs and worse outcomes which suggest - but cannot prove - causal relationships. Although causality is difficult to establish, at a minimum association studies could be harnessed to develop potentially helpful biomarkers. Therefore, a study which refined the definition of SDs to include on the extreme continuum a negative ultraslow potential validated that this ultraslow potential is the electrophysiological correlate of cortical infarction in patients. The authors go on to make the argument that the presence of SDs with the negative ultraslow potential could be used as a biomarker for infarction. In other words, SDs and their related phenomena could be a useful biomarker for neurological worsening [25].

\section{Potential SD-Mediated Causes of Injury}

Several clinical studies have sought to determine SDmediated mechanisms for worse outcomes following SAH. The primary mechanisms that have been implicated involve blood flow, oxygen availability, and altered brain metabolism after injury. The first of these studies determined the effect of cerebral blood flow on outcomes [26]. The normal hemodynamic response to an SD in humans without acute brain injury is hyperemia; however, in the setting of acute brain injury such as $\mathrm{SAH}$, investigators have found that an inverse, decreased blood flow response is also possible [26]. Such decreased blood flow could serve as a mechanism for ischemia and worse outcome following SAH [36]. For example, the study on duration of SDs discussed above also found that the longer the SD, the more likely it was that the SD would cause reduced blood flow and DCI [22]. A case report building on 


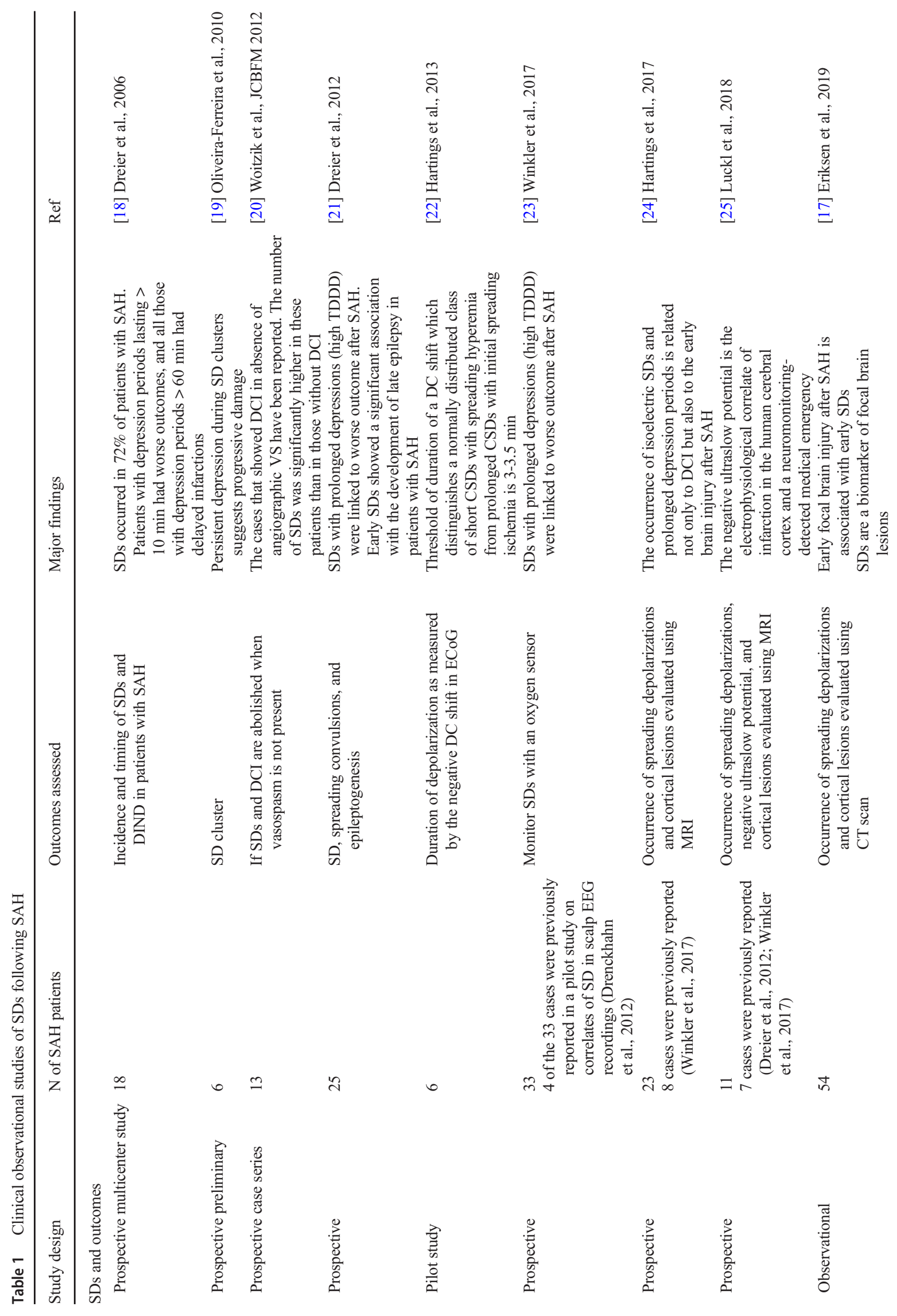




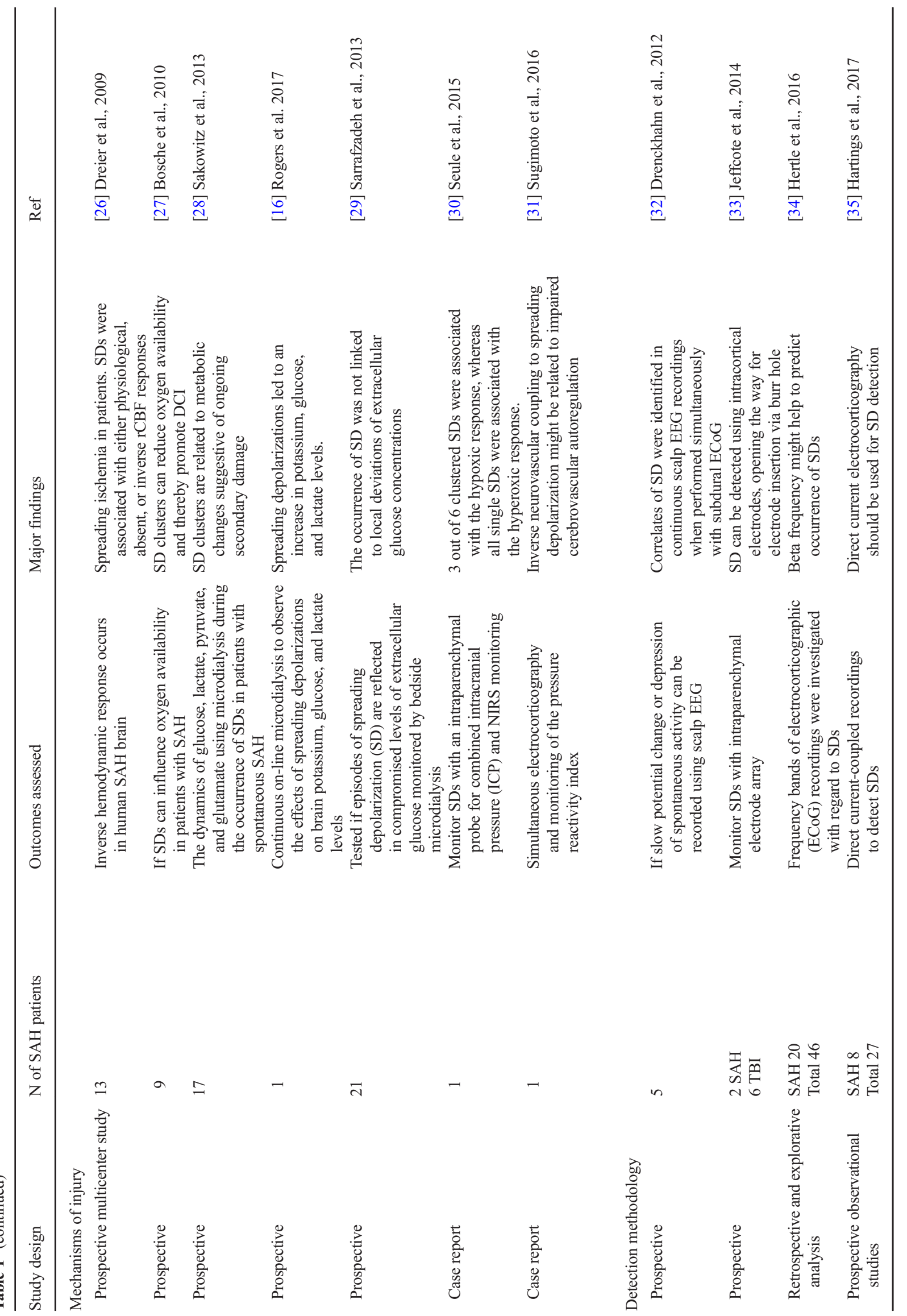


these findings used a continuous measure of cerebral autoregulation and found an association between impaired cerebrovascular regulation and increased duration of SD-related depressions [31].

Another potential mechanism of injury following SDs involves oxygen availability. In addition to decreasing blood flow, SDs can simultaneously increase oxygen demand through the energy required to reestablish ionic membrane gradients. Decreased oxygen supply and increased demand can have a marked effect on total oxygen availability [37]. A study using implanted cerebral oxygen sensors found that clusters of SDs can decrease oxygen availability in patients who go on to develop DCI [27]. Furthermore, investigators used near-infrared spectroscopy in a single case report and found that clustered SDs were associated with more hypoxic responses as compared to single SDs [30].

Finally, there is evidence that secondary injury following SDs may be mediated by metabolic changes in, for example, glucose and lactate levels. One study found significant changes in glucose and lactate levels, but not glutamate levels [28]. Another study involving 1 patient with nontraumatic SAH and using a novel continuous microdialysis method observed elevations in glucose and lactate levels during a presumptive SD, but did not assess for changes in glutamate [16]. Although there has been consistent evidence supporting the role of lactate changes, the evidence for the role of glucose metabolism remains controversial [29].

\section{Detection Methodology}

The gold standard in the clinical detection of SDs is to use direct current-coupled (DC) recordings from implanted subdural electrodes [35]. How to identify and diagnose SDs, and why SDs cannot be detected using certain amplifiers, have been addressed in consensus statements of the Co-Operative Studies on Brain Injury Depolarizations (COSBID) group [38]. Because DC recordings require specialized amplifiers that are not commonly available at most centers, 1 study examined the sensitivity of different intracranial electrocorticography (ECoG) frequency bands and found that modulation of the beta frequency might be predictive for the occurrence of an SD [34]. However, it is undoubtedly better to employ the correct DC amplifier or to disable the filter from AC/DC devices dedicated to evaluate epilepsy to better detect SDs in humans. Additional work will be needed to determine if beta frequency analysis will prove to be helpful in the detection of SDs.

The development of less invasive methods would also be helpful in expanding the number of centers that could detect SDs. When intracranial ECoG is recorded simultaneously with scalp EEG, correlates between the 2 modalities have been found [32]. However, it has not yet been possible to detect SDs with good-enough reliability with scalp EEG alone
[35]. Given these persistent challenges with surface EEG alone-without the benefit of intracranial recordingsinvestigators have developed methods to detect SDs with the use of an intraparenchymal depth electrode inserted through a burr hole [33]. Although still invasive, a depth electrode is arguably less invasive as it does not require a craniotomy and can be placed alongside other clinical probes (e.g., fiberoptic ICP and oxygenation monitors) through the same burr hole. A depth electrode also allows for SD monitoring after coil embolization. However, some might argue that depth electrodes are invasive in different ways as insertion requires penetration of the brain and microtrauma. Furthermore, the smaller cortical coverage offered by depth recording likely makes it less sensitive for SDs as compared to the broader coverage from subdural strip recording, as evidenced by a study demonstrating that $43 \%$ of SDs were not detected on all leads of a subdural strip [35].

\section{Animal Studies Involving SDs and SAH}

We found 20 papers on SD and SAH in animal models of the disease (Table 2). There were multiple species (e.g., cat, mouse, pig, and rat) and multiple different SAH induction methods employed (e.g., blood injection into various compartments and endovascular perforation). The most common species used was rat, and the most common induction model was a SAH-mimicking model which is described in more detail below. The animal studies can be roughly grouped by those that observe SDs in SAH models, explore a mechanism of injury, or test a therapeutic intervention.

\section{Observation of SAH and SDs in Animals}

The observational studies have established that SDs following SAH can be detected using electrocorticography (ECoG) [24, 39], extracellular potassium recording [40], MRI [41, 42, 44], and cerebral blood flow and NADH imaging [43]. These studies also determined the sequelae of SDs in the setting of SAH by infarct volume on histology, activity of the $\mathrm{Na}^{+} / \mathrm{K}^{+}$ATPase, and mortality and functional outcomes. Notably, experimental SDs have only been observed in the acute phase. In a study designed to assess delayed effects, no SDs were observed during an observation period lasting 3 days [45].

\section{Mechanistic Studies in Animals}

There have been several studies performed in rats which have explored potential SD-mediated mechanisms of injury following SAH. These studies have implicated nitric oxide (NO), extracellular potassium, neurovascular coupling, ischemia, ATPase activity, and calcium signaling in the pathogenesis 


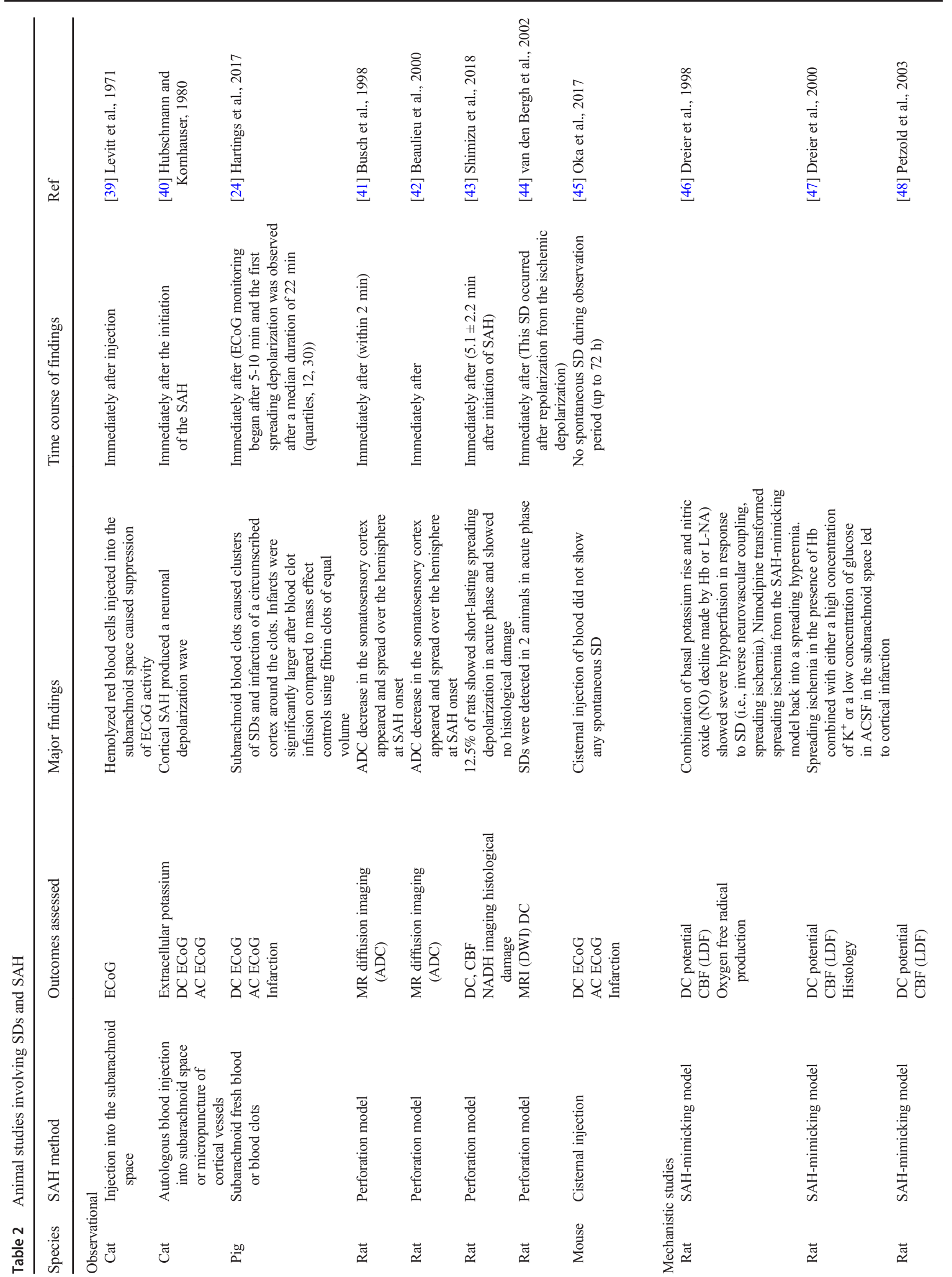




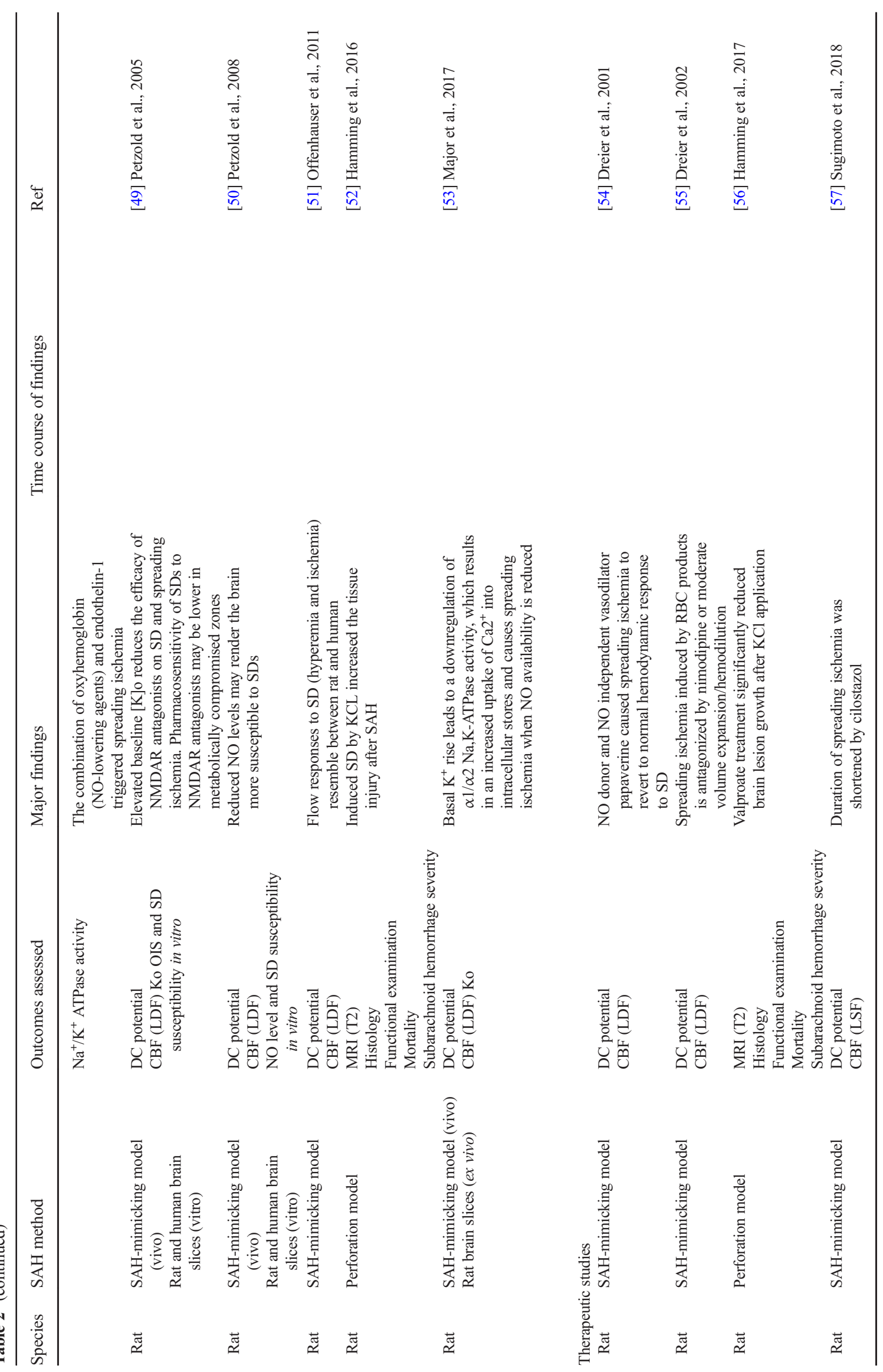


of injury. Experimental work has demonstrated that SDs can play a causal role. SDs induced with topical potassium chloride worsen tissue injury in a rat endovascular puncture model of SAH [52].

All of the remaining mechanistic knowledge comes from rat studies from the Berlin group using a SAH-mimicking model consisting of artificial CSF and purified hemoglobin with various levels of potassium that is superperfused in a closed cranial window preparation. An early study produced conditions that caused cortical spreading ischemia in rats-as an analogue to DCI after SAH in humans - by elevating extracellular potassium and decreasing NO availability [46]. This study also found that the spreading ischemia associated with the SAH model could be transformed to a spreading hyperemia with nimodipine. A follow-up study showed that these spreading ischemia events can lead to necrosis [47]. Building on this work, the group found that a potent endogenous vasoconstrictor, endothelin-1 (ET-1), potentiates NOmediated spreading ischemia through vasoconstriction and also through an effect on the sodium-potassium ATPase [48]. Furthermore, they found that elevated potassiumwhich can be present in the setting of SAH-decreases the efficacy of SD-inhibiting drugs such as NMDA receptor antagonists [49]. In subsequent studies utilizing rat and human tissue, endothelial NO production was implicated in determining thresholds for the presence of SD [50]. There was no difference in blood flow responses between rat and human brains [51]. Finally, the group found that spreading ischemia in their SAH-mimicking rat model could be mediated by the alpha1/alpha2 $\mathrm{Na} / \mathrm{K}$-ATPase and elevated intracellular calcium stores in multiple cell types [53].

\section{Therapeutic Studies in Animals}

Several therapeutic interventions targeting SDs have been tested in animal models, mostly focused on the mechanisms discussed above. The Berlin group demonstrated that spreading ischemia could be transformed to hyperemia using drugs that cause vasodilation through NO-dependent or NO-independent pathways [54]. They also showed that volume expansion with IV fluids and nimodipine, a calcium channel blocker used clinically to prevent DCI, could antagonize spreading ischemia through effects on the microcirculation [55]. In collaboration with other investigators in a clinical-translational paper, they found that the phosphodiesterase-3 inhibitor cilostazol could decrease the duration of spreading ischemia in their rat model of SAH and SDs [57]. Finally, a different group targeted SDs using valproate and found that valproate mitigated injury from induced SD in an endovascular perforation model of SAH [56]. The exact mechanism of valproate's effect was not clear as the number of SDs was not significantly altered. A therapeutic role for valproate remains a question for future study.

\section{Clinical Treatment Studies}

Despite associations seen in observational clinical studies and promising animal work, there have only been 2 prospective trials to date that targeted SDs to prevent secondary brain injury and improve outcomes in patients following SAH (Table 3). The other studies assessing potential therapies are retrospective. One of the retrospective studies measured the frequency of SDs in patients with acute brain injury, including SAH, and found that use of ketamine was associated with fewer SDs as compared to other sedatives [58]. Another retrospective study looked specifically at ketamine in patients requiring a higher level of sedation and found that the onset of ketamine infusion was associated with a decreased incidence of subsequent SDs [15]. A third retrospective study looked at home medications prior to hospital admission, determined if they fell into SD-promoting or SD-inhibiting drug categories, and found that SD-inhibiting drugs were associated with a reduction in DCI but had no effect on measured functional outcomes; however, this study could not determine the actual incidence of SDs in their patients [59]. A small prospective randomized multiple crossover study which included 8 patients with SAH found that ketamine inhibited SDs in critically ill intubated and nonintubated patients [60] but was not able to assess effects on functional outcomes. Finally, a randomized trial of $50 \mathrm{SAH}$ patients with subdural electrode monitoring found that the phosphodiesterase-3 inhibitor cilostazol led to a statistically significant decreased duration of SD-induced spreading depression times per day (adjusted $\beta=-252, p=$ 0.04 ) and a trend for a lower rate of DCI (odds ratio 0.27, $p=$ 0.08) [57]. Together with another randomized trial which found that cilostazol had a statistically significant decrease in poor functional outcome using a dichotomized Glascow outcome scale [61], these results are consistent with the idea that cilostazol improves functional outcome by mitigating the detrimental effects from SDs.

\section{Discussion}

Secondary injury remains an important contributor to worse functional outcomes following SAH. An association between SDs and worse injury in the setting of SAH has been well established, and therapies designed to target SDs have shown some promise in small randomized trials. Furthermore, SDs can cause worse tissue injury in animal models of SAH, and basic translational studies have been essential in developing novel therapies for SD-mediated injury after SAH. 


\section{Clinical Studies}

There is a strong association between spreading depolarizations and tissue and functional outcome in patients with aneurysmal SAH. Basic preclinical studies have provided enough evidence of potential causality to attempt translation in therapeutic studies involving patients with SAH. One of the most discussed therapies to target SDs has been the NMDA receptor antagonist ketamine, which decreases the incidence of SDs [62]. There is good retrospective evidence $[15,58]$ and limited prospective data [60] suggesting that ketamine has an effect on SD, but an adequately powered randomized trial assessing functional outcomes has yet to be performed. Another therapy that appears promising from rat studies is valproate [56]; however, there are no focused clinical studies that we could identify that reported the effect of valproate on clinical SD metrics or patient outcomes following SAH.

Cilostazol is a very promising SD-modulating agent [57, 61]. It is worth noting that the proposed mechanism of cilostazol is on a decrease in brain activity depression following an SD, rather than on the blockage of SDs themselves. In other words, cilostazol's effect appears to mitigate the sequela of SDs once they have already occurred, potentially through a mechanism involving cerebral blood flow [57]. A potential additional area of research is on the effect of cilostazol on neurovascular coupling. Major caveats to the cilostazol studies are that they were performed at single, albeit independent, centers. Furthermore, the studies were performed in Japan where the ROCK inhibitor fasudil is used prophylactically to prevent secondary injury, instead of the calcium channel inhibitor nimodipine which is used in most other countries. As with any targeted therapy, there is always the possibility that cilostazol is acting more directly on a factor that impacts outcome independent of SDs, and that SDs are an epiphenomenon. Given that patients improved with cilostazol and that a SD metric, duration of depression per SD, was identified that improved with treatment, SDs could - at a minimumrepresent an important biomarker for therapeutic response. However, a major barrier to the use of SDs as a biomarker is the need for specialized, invasive neuromonitoring and experienced teams to interpret the data [38]. Invasive detection either has to be made easier for wider adoption or a reliable noninvasive modality needs to be developed. Both solutions are not trivial.

There are a few potential studies that could build on the current progress made in the field. We would suggest that a multicenter randomized trial of cilostazol following $\mathrm{SAH}$ is warranted to validate the generalizability of the promising findings seen by the 2 independent Japanese studies. Performance of such a trial could be done without the requirement to detect SDs, because a benefit on functional outcomes following cilostazol was seen without the need to detect SDs. However, SDs could be assessed in a subset 
of centers already accustomed to the approach to better stratify injury severity or to potentially determine which patient populations would be most likely to benefit from treatment.

Randomized trials focused on SD inhibition are also warranted, especially because cilostazol does not inhibit SD itself. The most promising clinically used SD-blocking agent is ketamine [62]. Because good retrospective and pilot randomized studies have already been performed, it would make sense to proceed with a randomized trial of ketamine that is adequately powered to determine long-term functional patient outcomes. Finally, there is preclinical evidence of an effect of the anticonvulsant valproate on injury markers following $\mathrm{SAH}$, but no clinical studies reporting positive or negative results. Therefore, any study addressing the effect of valproate in the SAH patient population would fill gaps in our current state of knowledge.

\section{Mechanistic Studies}

Animal models that recapitulate SDs following SAH have been critical in assessing potential mechanisms and therapies for persistent injury following SAH. The animal studies to date ("Results" and Table 2) comprise the foundational work in the field. Much attention, in particular, has been spent addressing the role of potassium and NO after SDs and SAH. Rat studies using artificial CSF perfusates have indicated that potassium and NO both play a role in the incidence of SDs and affect cerebral blood flow and the degree of tissue injury. However, basic questions are still unanswered regarding, for example, their CSF concentrations in other models of SAH and in humans following SDs. It is worth mentioning that ischemia itself causes a gradual increase of interstitial potassium before it causes SD [63, 64]. Therefore, increased potassium concentration in the subarachnoid clot may not be necessary for SD and spreading ischemia after SAH. On the other hand, there is evidence that potassium may be elevated in the blood clot [65]. It is also worth noting that the synthesis of NO is oxygen-dependent $[66,67]$. This could contribute to the lack of $\mathrm{NO}$ after $\mathrm{SAH}$, in addition to many other factors [36, $68,69]$. Another general limitation to small animal models is that the subarachnoid space is much smaller than that of humans. Therefore, accumulation of erythrocyte products in the subarachnoid space is less likely in small animals than in humans.

There are other areas that require further study. Given the promising clinical cilostazol studies, we believe that additional preclinical studies - using complementary SAH models like endovascular perforation or blood injection-would be useful to better understand the potential causal role of SD on the effect of cilostazol, particularly regarding its effect on neurovascular coupling. It will also be important to further study the effect of SDs themselves in animal models to further delineate where they fall in the causal pathway. For example, the 1 study that showed that induced SDs cause worse tissue outcome using an endovascular perforation model in rats had to use an invasive SD-induction method requiring drilling of a burr hole and topical application of high-concentration $\mathrm{KCl}$ to the surface of the brain. Recently, investigators have developed an approach to induce SDs noninvasively in transgenic optogenetic mice [70]. It would be interesting to see if SDs cause additional brain injury and behavioral changes without the confounds from invasive induction techniques.

Another important question that animal studies may be able to address is why patients without frank brain injury following their hospitalization go on to have longstanding cognitive deficits. Human imaging studies suggest an association between measures of functional brain network connectivity and worse cognitive outcomes in survivors of aneurysm rupture [71, 72]. However, it is not clear if the cognitive deficits are determined by altered functional connectivity or if functional connectivity is an epiphenomenon. Recently, investigators have described a mouse model of subarachnoid hemorrhage in which resting state functional connectivity and neurocognitive behavior can be determined with relatively high throughput [73]. They found that $\mathrm{SAH}$ leads to specific deficits in functional connectivity and cognition, but did not assess the impact of SDs. SD not only causes spreading depression of activity but also spreading suppression of low-frequency vascular fluctuations - the signal on which resting state functional connectivity is based-in humans [26]. Therefore, there may be another interesting connection between functional connectivity and SD research. In future studies, it would be interesting to determine the effect of induced delayed SDs on injury, behavior, and functional connectivity in a mouse model of SAH.

The pitfalls of SAH animal models have been recently described extensively elsewhere [4]. In general, every animal model is limited because models necessarily require abstraction and deconstruction of the factors hypothesized to contribute to the pathophysiology of a disease. Dissecting individual factors, such as SAH, SD occurrence, and different concentrations of NO, potassium, and calcium in different tissue compartments, for example, enables the assessment of each factor to the whole. However, the process is intrinsically artificial, and, in any case, there are equally vexing problems with seemingly perfect models. For our purposes, it would be useful to have access to a model (which is nonexistent) with naturally developing aneurysms, reliable rupture rates, controllable injury severity, and the occurrence of spontaneous delayed SDs and delayed infarcts. The issue with such perfect models is that it becomes difficult to assess the contribution of the individual components. For example, if delayed SDs occur spontaneously, then a blocker needs to be applied to infer its contribution. However, any therapy can have potential off-target effects. NMDA receptor antagonists such as ketamine or MK801 can be used to block SDs but are also known to have 
neuroprotective effects independent of SDs. The other major problem with seemingly perfect models is that they are still models and may not lead to translation to humans simply by being of a different species.

Regardless, it is helpful to consider some of the advantages and limitations of the specific animal models used for SD and SAH studies. The SAH-mimicking model used by the Berlin group enables a great deal of control of the extracellular milieu and blood products. It is ideal for determining how specific factors can contribute to spontaneous SD occurrence. However, the model does not reproduce the elevated ICP seen in other SAH models. The endovascular perforation model causes elevated ICP analogous to that seen in patients, and it might be thought of as more natural than other models because an artery is being ruptured, as is the case in humans. However, endovascular perforation frequently leads to an ischemic stroke because of occlusion from the perforating filament. Not only does this complicate the model in that endovascular perforation really is a SAH plus acute ischemic stroke model, but ischemia is itself a strong inducer of SDs. Therefore, it is not possible to determine to what degree SDs are interacting with ischemia versus with SAH. Furthermore, the filament that is needed for perforation can also cause endothelial damage and thrombus formation that may not be analogous to what happens in patients. Also, the injury severity with perforation tends to be highly variable. There are blood injection models targeting anterior or posterior CSF spaces that get around some of these issues. The advantage to injection is that the rate of ICP rise-particularly in anterior injection models - and volume of blood can be well controlled, leading to titratable and more reliable injury severity. The disadvantage is that injection from a needle is undoubtedly artificial and needle insertion by itself can cause injury.

The SAH model issue is further compounded by how SDs are modeled. Should SDs develop spontaneously in a model or should they be induced in some way, for example, from potassium application, ischemia, or optogenetic stimulation? SD animal studies have only resulted in SDs observed in the acute phase, shortly after SAH was simulated. In all the animal models in which SDs are observed, they are only observed in the acute phase. The one study that looked for delayed SDs did not observe spontaneous SDs, but did find that SAH influences the frequency of delayed ischemia-induced SDs in an injection mouse model of SAH (Table 2) [45]. It is unknown why this is the case. However, it appears that the only way to currently determine the effect of delayed SDs in the lab is to induce them exogenously. It is not clear if this is a weakness or a strength. Does exogenous SD induction make the model unnatural, or does it allow for the abstraction needed to determine the contribution of SDs to brain injury? There is perhaps another promising experimental model system that has not yet been fully assessed. In the elastase model of SAH, aneurysms are induced with injection of elastase at the base of the brain with spontaneous aneurysm rupture within 2 weeks [74]. This could be a promising approach to complement existing SAH plus SD models.

Finally, it is notable that there are a limited number of active, independent groups studying the role of SDs in SAH patients or model systems. This may be due to high barriers for entry given the need for invasive electrophysiology or advanced imaging approaches to detect SDs. Barriers to enter the field have been lowered recently with validations of more convenient noninvasive SD detection modalities [75]. Furthermore, the Co-Operative Studies on Brain Injury Depolarizations (COSBID) consortium has been extraordinarily welcoming to new concepts and research groups [76]. However, there remains a continued need for a greater diversity of investigators and studies for the field to further advance.

\section{Additional Limitations}

This review is limited by its narrative nature, and there is a possibility that some studies not registered in Pubmed were not captured by our search. We had to exhibit some judgment in choosing the studies to include. Therefore, this paper should not be considered a comprehensive systematic review. Rather, we sought to perform a focused review and commentary on the current state of the field of SDs and SAH. We further narrowed our focus to manuscripts which studied both SAH and SD, although studies of SDs in other disease states could inform the role of SDs in SAH. This could be a goal of future reviews. Finally, many of the reviewed manuscripts include studies with less than 25 subjects which indicates that additional, larger studies are sorely needed for further progress in the field.

\section{Conclusion}

There is compelling evidence that SDs are associated with worse physiological and functional outcomes in patients with SAH. At a minimum, this translates to SDs being a potentially useful biomarker for injury severity. Clinical trials and preclinical animal work continue to be urgently needed to determine where SD lies in the causal pathway of further brain damage following SAH and, most importantly, to develop therapies to improve patient outcomes.

Acknowledgments We would like to thank the peer reviewers who provided very helpful comments and the support and expertise of the CoOperative Studies on Brain Injury Depolarizations (COSBID) community in shaping many of the questions and approaches raised in our manuscript. This work was funded by the National Institutes of Health (R25NS065743, KL2TR002542, and K08NS112601); the American Heart Association and American Stroke Association (18POST34030369); the Andrew David Heitman Foundation; the Aneurysm and AVM Foundation; and the Brain Aneurysm Foundation's Timothy P. Susco and Andrew David Heitman Foundation Chairs of Research. 
Required Author Forms Disclosure forms provided by the authors are available with the online version of this article.

\section{Glossary: Modified from Dreier et al. [38]}

Spreading depolarization

SD cluster

Negative DC shift

Negative ultraslow potential

\section{References}

1. Maher M, Schweizer TA, Macdonald RL. Treatment of Spontaneous Subarachnoid Hemorrhage: Guidelines and Gaps. Stroke. 2020;STROKEAHA119025997.
2. Rass V, Helbok R. Early Brain Injury After Poor-Grade Subarachnoid Hemorrhage. Curr Neurol Neurosci Rep. 2019;19(10):78.

3. Vergouwen MD, Vermeulen M, van Gijn J, Rinkel GJ, Wijdicks EF, Muizelaar JP, et al. Definition of delayed cerebral ischemia after aneurysmal subarachnoid hemorrhage as an outcome event in clinical trials and observational studies: proposal of a multidisciplinary research group. Stroke. 2010;41(10):2391-2395.

4. Oka F, Chung DY, Suzuki M, Ayata C. Delayed Cerebral Ischemia After Subarachnoid Hemorrhage: Experimental-Clinical Disconnect and the Unmet Need. Neurocritical care. 2019.

5. Terpolilli NA, Brem C, Buhler D, Plesnila N. Are We Barking Up the Wrong Vessels? Cerebral Microcirculation After Subarachnoid Hemorrhage Stroke. 2015;46(10):3014-3019.

6. Chung DY, Oka F, Ayata C. Spreading Depolarizations: A Therapeutic Target Against Delayed Cerebral Ischemia After Subarachnoid Hemorrhage. J Clin Neurophysiol. 2016;33(3): 196-202.

7. Ayata C, Lauritzen M. Spreading Depression, Spreading Depolarizations, and the Cerebral Vasculature. Physiol Rev. 2015;95(3):953-993.

8. Dreier JP, Reiffurth C. The stroke-migraine depolarization continuum. Neuron. 2015;86(4):902-922.

9. Major S, Huo S, Lemale CL, Siebert E, Milakara D, Woitzik J, et al. Direct electrophysiological evidence that spreading depolarizationinduced spreading depression is the pathophysiological correlate of the migraine aura and a review of the spreading depolarization continuum of acute neuronal mass injury. Geroscience. 2020;42(1):57-80.

10. Dreier JP, Lemale CL, Kola V, Friedman A, Schoknecht K. Spreading depolarization is not an epiphenomenon but the principal mechanism of the cytotoxic edema in various gray matter structures of the brain during stroke. Neuropharmacology. 2018;134(Pt B): 189-207.

11. Gursoy-Ozdemir Y, Qiu J, Matsuoka N, Bolay H, Bermpohl D, Jin $\mathrm{H}$, et al. Cortical spreading depression activates and upregulates MMP-9. J Clin Invest. 2004;113(10):1447-1455.

12. Sadeghian H, Lacoste B, Qin T, Toussay X, Rosa R, Oka F, et al. Spreading depolarizations trigger caveolin-1-dependent endothelial transcytosis. Ann Neurol. 2018;84(3):409-423.

13. Lublinsky S, Major S, Kola V, Horst V, Santos E, Platz J, et al. Early blood-brain barrier dysfunction predicts neurological outcome following aneurysmal subarachnoid hemorrhage. EBioMedicine. 2019;43:460-472.

14. Mestre H, Du T, Sweeney AM, Liu G, Samson AJ, Peng W, et al. Cerebrospinal fluid influx drives acute ischemic tissue swelling. Science. 2020.

15. Santos E, Olivares-Rivera A, Major S, Sanchez-Porras R, Uhlmann L, Kunzmann K, et al. Lasting s-ketamine block of spreading depolarizations in subarachnoid hemorrhage: a retrospective cohort study. Crit Care. 2019;23(1):427.

16. Rogers ML, Leong CL, Gowers SA, Samper IC, Jewell SL, Khan A, et al. Simultaneous monitoring of potassium, glucose and lactate during spreading depolarization in the injured human brain - Proof of principle of a novel real-time neurochemical analysis system, continuous online microdialysis. J Cereb Blood Flow Metab 2017;37(5):1883-1895.

17. Eriksen N, Rostrup E, Fabricius M, Scheel M, Major S, Winkler MKL, et al. Early focal brain injury after subarachnoid hemorrhage correlates with spreading depolarizations. Neurology 2019;92(4): e326-e341.

18. Dreier JP, Woitzik J, Fabricius M, Bhatia R, Major S, Drenckhahn $\mathrm{C}$, et al. Delayed ischaemic neurological deficits after subarachnoid haemorrhage are associated with clusters of spreading depolarizations. Brain J Neurol 2006;129(Pt 12):3224-3237. 
19. Oliveira-Ferreira AI, Milakara D, Alam M, Jorks D, Major S, Hartings JA, et al. Experimental and preliminary clinical evidence of an ischemic zone with prolonged negative DC shifts surrounded by a normally perfused tissue belt with persistent electrocorticographic depression. J Cereb Blood Flow Metab. 2010;30(8):1504-1519.

20. Woitzik J, Dreier JP, Hecht N, Fiss I, Sandow N, Major S, et al. Delayed cerebral ischemia and spreading depolarization in absence of angiographic vasospasm after subarachnoid hemorrhage. J Cereb Blood Flow Metab 2012;32(2):203-212.

21. Dreier JP, Major S, Pannek HW, Woitzik J, Scheel M, Wiesenthal $\mathrm{D}$, et al. Spreading convulsions, spreading depolarization and epileptogenesis in human cerebral cortex. Brain: J Neurol. 2012;135(Pt 1):259-275.

22. Hartings JA, Wilson JA, Look AC, Vagal A, Shutter LA, Dreier JP, et al. Full-band electrocorticography of spreading depolarizations in patients with aneurysmal subarachnoid hemorrhage. Acta Neurochir Suppl 2013;115:131-141.

23. Winkler MK, Dengler N, Hecht N, Hartings JA, Kang EJ, Major S, et al. Oxygen availability and spreading depolarizations provide complementary prognostic information in neuromonitoring of aneurysmal subarachnoid hemorrhage patients. J Cereb Blood Flow Metab. 2017;37(5):1841-1856.

24. Hartings JA, York J, Carroll CP, Hinzman JM, Mahoney E, Krueger $\mathrm{B}$, et al. Subarachnoid blood acutely induces spreading depolarizations and early cortical infarction. Brain: J Neurol 2017;140(10): 2673-2690.

25. Luck1 J, Lemale CL, Kola V, Horst V, Khojasteh U, OliveiraFerreira AI, et al. The negative ultraslow potential, electrophysiological correlate of infarction in the human cortex. Brain: J Neurol 2018;141(6):1734-1752.

26. Dreier JP, Major S, Manning A, Woitzik J, Drenckhahn C, Steinbrink J, et al. Cortical spreading ischaemia is a novel process involved in ischaemic damage in patients with aneurysmal subarachnoid haemorrhage. Brain: J Neurol. 2009;132(Pt 7):18661881.

27. Bosche B, Graf R, Ernestus RI, Dohmen C, Reithmeier T, Brinker $\mathrm{G}$, et al. Recurrent spreading depolarizations after subarachnoid hemorrhage decreases oxygen availability in human cerebral cortex. Ann Neurol. 2010;67(5):607-617.

28. Sakowitz OW, Santos E, Nagel A, Krajewski KL, Hertle DN, Vajkoczy P, et al. Clusters of spreading depolarizations are associated with disturbed cerebral metabolism in patients with aneurysmal subarachnoid hemorrhage. Stroke. 2013;44(1):220-223.

29. Sarrafzadeh A, Santos E, Wiesenthal D, Martus P, Vajkoczy P, Oehmchen M, et al. Cerebral glucose and spreading depolarization in patients with aneurysmal subarachnoid hemorrhage. Acta Neurochir Suppl. 2013;115:143-147.

30. Seule M, Keller E, Unterberg A, Sakowitz O. The Hemodynamic Response of Spreading Depolarization Observed by Near Infrared Spectroscopy After Aneurysmal Subarachnoid Hemorrhage. Neurocrit Care. 2015;23(1):108-112.

31. Sugimoto K, Shirao S, Koizumi H, Inoue T, Oka F, Maruta Y, et al. Continuous Monitoring of Spreading Depolarization and Cerebrovascular Autoregulation after Aneurysmal Subarachnoid Hemorrhage. J Stroke Cereb Dis. 2016;25(10):e171-e177.

32. Drenckhahn C, Winkler MK, Major S, Scheel M, Kang EJ, Pinczolits A, et al. Correlates of spreading depolarization in human scalp electroencephalography. Brain: J Neurol. 2012;135(Pt 3): $853-868$.

33. Jeffcote T, Hinzman JM, Jewell SL, Learney RM, Pahl C, Tolias C, et al. Detection of spreading depolarization with intraparenchymal electrodes in the injured human brain. Neurocrit Care. 2014;20(1): 21-31.

34. Hertle DN, Heer M, Santos E, Scholl M, Kowoll CM, Dohmen C, et al. Changes in electrocorticographic beta frequency components precede spreading depolarization in patients with acute brain injury. Clin Neurophysiol. 2016;127(7):2661-2667.

35. Hartings JA, Li C, Hinzman JM, Shuttleworth CW, Ernst GL, Dreier JP, et al. Direct current electrocorticography for clinical neuromonitoring of spreading depolarizations. J Cereb Blood Flow Metab. 2017;37(5):1857-1870.

36. Dreier JP. The role of spreading depression, spreading depolarization and spreading ischemia in neurological disease. Nature medicine. 2011;17(4):439-447.

37. von Bornstadt D, Houben T, Seidel JL, Zheng Y, Dilekoz E, Qin T, et al. Supply-demand mismatch transients in susceptible peri-infarct hot zones explain the origins of spreading injury depolarizations. Neuron. 2015;85(5):1117-1131.

38. Dreier JP, Fabricius M, Ayata C, Sakowitz OW, William Shuttleworth C, Dohmen C, et al. Recording, analysis, and interpretation of spreading depolarizations in neurointensive care: Review and recommendations of the COSBID research group. J Cereb Blood Flow Metab. 2017;37(5):1595-1625.

39. Levitt P, Wilson WP, Wilkins RH. The effects of subarachnoid blood on the electrocorticogram of the cat. J Neurosurg. 1971;35(2):185-191.

40. Hubschmann OR, Kornhauser D. Cortical cellular response in acute subarachnoid hemorrhage. J Neurosurg. 1980;52(4):456-462.

41. Busch E, Beaulieu C, de Crespigny A, Moseley ME. Diffusion MR imaging during acute subarachnoid hemorrhage in rats. Stroke. 1998;29(10):2155-2161.

42. Beaulieu C, Busch E, de Crespigny A, Moseley ME. Spreading waves of transient and prolonged decreases in water diffusion after subarachnoid hemorrhage in rats. Magn Reson Med. 2000;44(1): 110-116.

43. Shimizu T, Hishikawa T, Nishihiro S, Shinji Y, Takasugi Y, Haruma $\mathrm{J}$, et al. NADH fluorescence imaging and the histological impact of cortical spreading depolarization during the acute phase of subarachnoid hemorrhage in rats. J Neurosurg. 2018;128(1):137-143.

44. van den Bergh WM, Zuur JK, Kamerling NA, van Asseldonk JT, Rinkel GJ, Tulleken CA, et al. Role of magnesium in the reduction of ischemic depolarization and lesion volume after experimental subarachnoid hemorrhage. J Neurosurg. 2002;97(2):416-422.

45. Oka F, Hoffmann U, Lee JH, Shin HK, Chung DY, Yuzawa I, et al. Requisite ischemia for spreading depolarization occurrence after subarachnoid hemorrhage in rodents. J Cereb Blood Flow Metab. 2017;37(5):1829-1840.

46. Dreier JP, Korner K, Ebert N, Gorner A, Rubin I, Back T, et al. Nitric oxide scavenging by hemoglobin or nitric oxide synthase inhibition by N-nitro-L-arginine induces cortical spreading ischemia when $\mathrm{K}+$ is increased in the subarachnoid space. J Cereb Blood Flow Metab. 1998;18(9):978-990.

47. Dreier JP, Ebert N, Priller J, Megow D, Lindauer U, Klee R, et al. Products of hemolysis in the subarachnoid space inducing spreading ischemia in the cortex and focal necrosis in rats: a model for delayed ischemic neurological deficits after subarachnoid hemorrhage? J Neurosurg. 2000;93(4):658-666.

48. Petzold GC, Einhaupl KM, Dirnagl U, Dreier JP. Ischemia triggered by spreading neuronal activation is induced by endothelin-1 and hemoglobin in the subarachnoid space. Ann Neurol. 2003;54(5): 591-598.

49. Petzold GC, Windmuller O, Haack S, Major S, Buchheim K, Megow D, et al. Increased extracellular $\mathrm{K}+$ concentration reduces the efficacy of N-methyl-D-aspartate receptor antagonists to block spreading depression-like depolarizations and spreading ischemia. Stroke. 2005;36(6):1270-1277.

50. Petzold GC, Haack S, von Bohlen Und Halbach O, Priller J, Lehmann TN, Heinemann U, et al. Nitric oxide modulates spreading depolarization threshold in the human and rodent cortex. Stroke. 2008;39(4):1292-1299. 
51. Offenhauser N, Windmuller O, Strong AJ, Fuhr S, Dreier JP. The gamut of blood flow responses coupled to spreading depolarization in rat and human brain: from hyperemia to prolonged ischemia. Acta Neurochir Suppl 2011;110(Pt 1):119-124.

52. Hamming AM, Wermer MJH, Umesh Rudrapatna S, Lanier C, van Os HJA, van den Bergh WM, et al. Spreading depolarizations increase delayed brain injury in a rat model of subarachnoid hemorrhage. J Cereb Blood Flow Metab. 2016;36(7):1224-1231.

53. Major S, Petzold GC, Reiffurth C, Windmuller O, Foddis M, Lindauer $\mathrm{U}$, et al. A role of the sodium pump in spreading ischemia in rats. J Cereb Blood Flow Metab. 2017;37(5):1687-1705.

54. Dreier JP, Petzold G, Tille K, Lindauer U, Arnold G, Heinemann U, et al. Ischaemia triggered by spreading neuronal activation is inhibited by vasodilators in rats. J Physiol. 2001;531(Pt 2):515526.

55. Dreier JP, Windmuller O, Petzold G, Lindauer U, Einhaupl KM, Dirnagl U. Ischemia triggered by red blood cell products in the subarachnoid space is inhibited by nimodipine administration or moderate volume expansion/hemodilution in rats. Neurosurgery. 2002;51(6):1457-1465; discussion 65-7.

56. Hamming AM, van der Toorn A, Rudrapatna US, Ma L, van Os HJ, Ferrari MD, et al. Valproate Reduces Delayed Brain Injury in a Rat Model of Subarachnoid Hemorrhage. Stroke. 2017;48(2):452-458.

57. Sugimoto K, Nomura S, Shirao S, Inoue T, Ishihara H, Kawano R, et al. Cilostazol decreases duration of spreading depolarization and spreading ischemia after aneurysmal subarachnoid hemorrhage. Ann Neurol. 2018;84(6):873-885.

58. Hertle DN, Dreier JP, Woitzik J, Hartings JA, Bullock R, Okonkwo DO, et al. Effect of analgesics and sedatives on the occurrence of spreading depolarizations accompanying acute brain injury. Brain: J Neurol 2012;135(Pt 8):2390-2398.

59. Hamming AM, Mulder IA, Gathier CS, van den Bergh WM, Dankbaar JW, Hoff RG, et al. Spreading depolarizationmodulating drugs and delayed cerebral ischemia after subarachnoid hemorrhage: A hypothesis-generating retrospective clinical study. J Neurol Sci. 2016;366:224-228.

60. Carlson AP, Abbas M, Alunday RL, Qeadan F, Shuttleworth CW. Spreading depolarization in acute brain injury inhibited by ketamine: a prospective, randomized, multiple crossover trial. J Neurosurg. 2018;1-7.

61. Matsuda N, Naraoka M, Ohkuma H, Shimamura N, Ito K, Asano $\mathrm{K}$, et al. Effect of Cilostazol on Cerebral Vasospasm and Outcome in Patients with Aneurysmal Subarachnoid Hemorrhage: A Randomized, Double-Blind, Placebo-Controlled Trial. Cerebrovasc Dis (Basel, Switzerland). 2016;42(1-2):97-105.

62. Helbok R, Hartings JA, Schiefecker A, Balanca B, Jewel S, Foreman B, et al. What Should a Clinician Do When Spreading Depolarizations are Observed in a Patient? Neurocr Care. 2019.

63. Erdemli G, Xu YZ, Krnjevic K. Potassium conductance causing hyperpolarization of CA1 hippocampal neurons during hypoxia. J Neurophysiol. 1998;80(5):2378-2390.
64. Muller M, Somjen GG. $\mathrm{Na}(+)$ and $\mathrm{K}(+)$ concentrations, extra- and intracellular voltages, and the effect of TTX in hypoxic rat hippocampal slices. J Neurophysiol. 2000;83(2):735-745.

65. Ohta O, Osaka K, Siguma M, Yamamoto M, Shimizu K, Toda N. Cerebral vasospasm following ruptured intracranial aneurysms, especially some contributions of potassium ion released from subarachnoid hematoma to delayed cerebral vasospasm. In: JA Bevan, editor. Vascular neuroeffector mechanisms. New York: Raven Press; 1983. p. 353-358.

66. Jiang HB, Yoneyama H, Furukawa A, Hamamoto T, Takahara J, Ichikawa Y. Effect of isosorbide dinitrate on nitric oxide synthase under hypoxia. Pharmacology 2001;62(1):10-16.

67. Uetsuka S, Fujisawa H, Yasuda H, Shima H, Suzuki M. Severe cerebral blood flow reduction inhibits nitric oxide synthesis. J Neurotrauma 2002;19(9):1105-1116.

68. Pluta RM, Hansen-Schwartz J, Dreier J, Vajkoczy P, Macdonald RL, Nishizawa $S$, et al. Cerebral vasospasm following subarachnoid hemorrhage: time for a new world of thought. Neurol Res. 2009;31(2):151-158.

69. Sakowitz OW, Wolfrum S, Sarrafzadeh AS, Stover JF, Dreier JP, Dendorfer A, et al. Relation of cerebral energy metabolism and extracellular nitrite and nitrate concentrations in patients after aneurysmal subarachnoid hemorrhage. J Cereb Blood Flow Metab. 2001;21(9):1067-1076.

70. Chung DY, Sadeghian H, Qin T, Lule S, Lee H, Karakaya F, et al. Determinants of Optogenetic Cortical Spreading Depolarizations. Cerebral Cortex (New York, NY : 1991). 2019;29(3):1150-1161.

71. da Costa L, Dunkley BT, Bethune A, Robertson A, Keller A, Pang EW. Increased Frontal Lobe Activation After Aneurysmal Subarachnoid Hemorrhage. Stroke. 2016.

72. Maher M, Churchill NW, de Oliveira Manoel AL, Graham SJ, Macdonald RL, Schweizer TA. Altered Resting-State Connectivity within Executive Networks after Aneurysmal Subarachnoid Hemorrhage. PloS One 2015;10(7):e0130483.

73. Chung DY, Oka F, Jin G, Harriott A, Kura S, Aykan S, et al. Subarachnoid hemorrhage leads to early and persistent functional connectivity and behavioral changes in mice. bioRxiv. $2019 ; 826891$.

74. Suzuki T, Takizawa T, Kamio Y, Qin T, Hashimoto T, Fujii Y, et al. Noninvasive Vagus Nerve Stimulation Prevents Ruptures and Improves Outcomes in a Model of Intracranial Aneurysm in Mice. Stroke. 2019;50(5):1216-1223.

75. Chung DY, Sugimoto K, Fischer P, Bohm M, Takizawa T, Sadeghian H, et al. Real-time non-invasive in vivo visible light detection of cortical spreading depolarizations in mice. J Neurosci Methods. 2018;309:143-146.

76. Shuttleworth CW, Andrew RD, Akbari Y, Ayata C, Balu R, Brennan KC, et al. Which Spreading Depolarizations Are Deleterious To Brain Tissue? Neurocrit Care. 2019.

Publisher's Note Springer Nature remains neutral with regard to jurisdictional claims in published maps and institutional affiliations. 